\title{
Associação da pressão arterial diastólica com o tempo acumulado de trabalho entre motoristas e cobradores
}

\section{Diastolic blood pressure and its association with cumulative working time among urban bus workers}

\author{
Ricardo Cordeiro*, Euclydes C. Lima Filho**, Frida M. Fischer" ${ }^{\star \star *}$, Djalma C. Moreira Filho*k*
}

\begin{abstract}
CORDEIRO, R. et al. Associação da pressão arterial diastólica com o tempo acumulado de trabalho entre motoristas e cobradores. Rev. Saúde Pública, 27: 363-72, 1993. Pela técnica de regressão linear múltipla, estudou-se a relação existente entre pressão arterial diastólica e as variáveis "tempo total acumulado de trabalho como condutor de veículos coletivos urbanos" e "idade", em uma população de 839 motoristas e cobradores, usuários de um serviço de saúde ocupacional da cidade de Campinas, Estado de São Paulo (Brasil). Os principais resultados encontrados foram associação positiva entre a pressão arterial diasiólica e o tempo acumulado de trabalho, bem como existência de uma interação entre esta variável e a idade dos condutores.
\end{abstract}

Descritores: Pressão arterial. Transportes. Trabalhadores.

\section{Introdução}

Tradicionalmente, os estudos epidemiológicosque envolvem populaçðes de trabalhadores têm se ocupado em pesquisar associações plausíveis, do ponto de vista fisiopatológico, entre patologias e supostos fatores de risco (Cordeiro $\left.{ }^{3}, 1991\right)$. Particularmente no estudo da hipertensão arterial sistêmica, é vasta a produção (Drettner ${ }^{5}, 1975$; Netterstrom \&Juel ${ }^{18}, 1988$; Kristensen ${ }^{13}$, 1989; Mackie \& O'Hanlon ${ }^{15}$, 1977; Shekelle e col. ${ }^{22}$, 1969; Talbot e col. ${ }^{23}$, 1985; Rusconi e col. ${ }^{21}$, 1975; Wu e col. $^{24}, 1987$; Carter $^{1}, 1980$ ). É também considerável a literatura que prioriza o estudo das implicações da organização do trabalho (Gardell e col. ${ }^{7}, 1977$; Karasek e col. ${ }^{11}$, 1979; Karasek $^{10}$, 1981; Karasek e col. ${ }^{12}$, 1982) e do "estresse ocupacional" (Cobb \& Rose², 1973; Cottington e col. ${ }^{4}, 1983$; Fouriad e col. $\left.{ }^{6}, 1984\right)$, na gênese da hipertensão arterial sistêmica.

Em 1987, Laurell \& Noriega ${ }^{14}$, ao estudarem a

* Departamento de Saúde Pública da Faculdade de Medicina de Botucatu (UNESP) - Botucatu, SP - Brasil

** Departamento de Estatística do Instituto de Matemática, Estatística e Ciências da Computação da UNICAMP Campinas, SP - Brasil

*** Departamento de Saúde Ambiental da Faculdade de Saúde Pública da Universidade de São Paulo - São Paulo - Brasil

**** Departamento de Medicina Preventiva e Social da Faculdade de Ciências Médicas da UNICAMP - Campinas, $\mathrm{SP}$ - Brasil

Separatas/Reprints: R. Cordeiro - Campus de Botucatu, $\mathrm{s} / \mathrm{n}^{\mathrm{o}}$ - 18618-000 - Botucatu, SP - Brasil

Edição subvencionada pela FAPESP. Processo Medicina 93/0208-5 distribuição de doenças entre operários de uma siderúrgica, notaram que estas se relacionavam não particularizadamente a um ou outro risco específico, mas sim a uma composição deles. Além disso, os autores estratificaram os trabalhadores de acordo com o tempo de trabalho na siderúrgica, notando que os grupos com maior tempo de trabalhoapresentavam umaprevalência significativamente maior de várias patologias. Esta metodologia parece interessante como uma abordagem preliminar na investigação das relações que se estabelecem entre a doença e o trabalho.

Avançando por este caminho, procurou-se no presente estudo distanciar-se da tradicional busca de associações entre doenças profíssionais e fatores de risco mais ou menos específicos, optando-se por uma abordagem um pouco mais genérica.

O objetivo da presente pesquisa foi estudar eventual associação entre a hipertensão arterial sistêmica e não um especificado fator de risco, mas sim o tempo acumulado de trabalho na categoria profissional de motoristas e cobradores do transporte coletivo urbano.

\section{Material e Método}

A população estudada foi constituída por motoristas e cobradores de veículos coletivos urbanos da cidade de Campinas (Estado de São Paulo, Brasil), usuários do Ambulatório de Saúde Ocupacional do Programa de Saúde do Trabalhador (PST), da citada cidade.

O PST, pelo seu ambulatório, presta atendimento em saúde ocupacional por intermédio de uma equipe multidisciplinar e alguns recursos técnicos especializa- 
dos. A demanda desse ambulatório é, em sua maior parte (cercade 70\%), encaminhada pelos sindicatos que fazem parte da comissão gerenciadora do PST. O restante é demanda referida pela rede pública primária de assistência à saúde, e demanda espontânea.

Para a execução do presente estudo foram analisados os prontuários dos 1.069 condutores urbanos de Campinas que se utilizaram do ambulatório médico do PST desde sua inauguração, em fevereiro de 1987 , até março de 1990. Os prontuários foram avaliados quanto à qualidade dasinformaçõesnelescontidas a respeito da investigação clínica da hipertensão arterial sistêmica (HAS) e classificados em duas categorias mutuamente exclusivas: "completo" e "incompleto"*. Os prontuários incompletos pertencentes acondutores, $108(10,1 \%$ da população inicialmente relacionada), foram descartados. Os 961 condutores restantes, cujos prontuários eram "completos", foram então classificados, quanto à funçãoque exerciam, em 3 categorias: motoristas (501), cobradores (338) e "outras funções"** (122), sendo esta última excluída do estudo. A população estudada foi, portanto, composta por 839 trabalhadores: 501 motoristas e 338 cobradores.

A variável "tempo de trabalho como condutor geral" (TCG) foi definida, para a população estudada como sendo a somatória do tempo, medida em meses, em que o indivíduo trabalhou nesta categoria em toda a sua vida laboral até o momento da primeira avaliação clínica realizada no PST.

Osindicato da categoria, "Sindicato dos Condutores de Veículos Rodoviários e Anexos", classifica o trabalho de seus representados em duas modalidades básicas assim denominadas: "urbano" e "frete".

O trabalho "urbano" é aquele desenvolvido nas empresas de transporte coletivo urbano de passageiros, que cobrem a malha viária urbana da cidade das $4,30 \mathrm{~h}$ da madrugada até às $2 h$ da madrugada seguinte. É exercido por motoristas e cobradores, que rodiziam em três turnos. Não existe uma regra bem definida para a formação das escalas de trabalho. Estas são rodiziadas entre os trabalhadores mês a mês.

Assim, para isolar-se o tempo de trabalho exclusivamente em veículos do tipo "transporte coletivo urbano de passageiros", definiu-se a variável "tempo de trabalho como condutor urbano" (TCU) como sendo

* Foram considerados incompletos os prontuários cujas anotações referentes à primeira avaliação clínica não continham (ou continham de modo incompleto) as informações referentes às aferições da pressão arterial sistêmica do condutor.

** Entre os condutores classificados em "outras funções" estavam todos aqueles trabalhadores necessários a uma empresa de transporte coletivo que não os motoristas e cobradores, tais como mecânicos, manobristas, faxineiros, escriturários, etc. A categoria dos condutores era composta, em março de 1990 , por cerca de 2.800 motoristas e cobradores. a somatória de todo o tempo, medida em meses, em que o indivíduo trabalhou exclusivamente no transporte de tipo"urbano", atê a sua primeira avaliação clínica no PST.

O transporte coletivo urbano de passageiros é utilizado, predominantemente, pelos trabalhadores de menor qualificação das pequenase médias empresas da cidade. Fugindo de sua dependência, as empresas de maior porte, em quase sua totalidade, desenvolveram um sistema próprio de transporte de seus empregados, o "frete".

O trabalho do tipo "frete" é exercido tipicamente por motoristas (não há cobradores nessa modalidade) em ônibus exclusivamente fretados pelas grandes empresas, para o transporte de seus empregados na rota "moradia-empresa-moradia".

A variável "tempo de trabalho como condutor frete" (TCF), foi definida como sendo a diferença, para cada trabalhador da população estudada, entre a TCG e a TCU (TCF = TCG - TCU) e, portanto, expressa o tempo de trabalho do condutor nas empresas do tipo "frete".

Uma vez que é muito grande a rotatividade de mãode-obra nesse setor, os motoristas em poucos anos de trabalho costumam passar por várias empresas; quer de transporte coletivo urbano de passageiros, quer de transporte coletivo fretadas. A título de exemplo, se um motorista trabalhou dois anos em uma empresa de transporte coletivo urbano de passageiros e um ano em uma empresa de transporte coletivo fretada, ele terá $\mathrm{TCG}=36$ meses, $\mathrm{TCU}=24$ meses e $\mathrm{TCF}=12$ meses. A variável TCF assume sempre o valor zero para os cobradores uma vez que para estes não existe a possibilidade de trabalho fora de empresas de transporte coletivo urbano de passageiros.

Para cada um dos trabalhadores componentes da população estudada foi coletado do prontuário médico o seguinte conjunto de dados, relativos à primeira avaliação clínica de cada trabalhador em questão: a) Idade (anos); b) TCG (meses); c) TCU (meses); d) TCF (meses); e) Pressão arterial diastólica (mmHg).

As Figuras 1 a 5 ilustram a distribuição das variáveis na população estudada.

Para o cômputo das variáveis TCG, TCU E TCF utilizaram-se as anotações relativas à história ocupacional. Estas são sistematicamente anotadas no prontuário quando da realização da "pré-consulta", a partir de relato dos próprios trabalhadores e análise minuciosa da carteira profissional dos mesmos.

Os dados relativos à pressão arterial diastólica (PAd) foram anotados de acordo com o seguinte procedimento: durante a primeira avaliação clínica, a pressão arterial do trabalhador foi aferida três vezes, pelo médico consultante, com esfigmomanômetro de coluna de mercúrio, respeitando um intervalo mínimo de $5 \mathrm{~min}$ entre as aferições. Quando estas medidas foram diferentes, anotou-se a menor entre elas. 


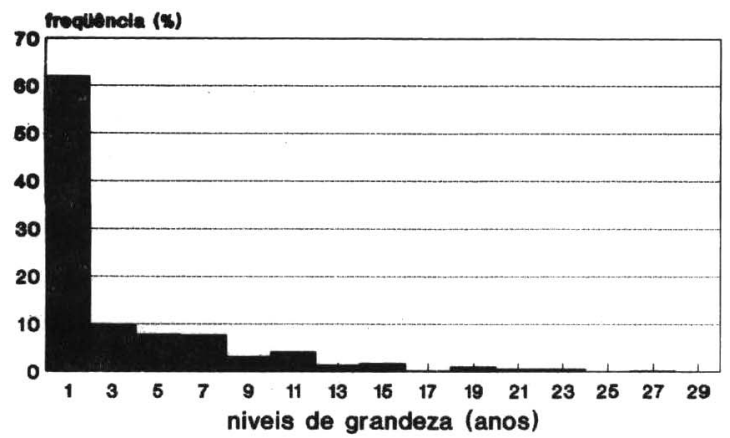

Figura 1. Dıstrıbulção de frequéncıa da ıdade na populaçào estudada

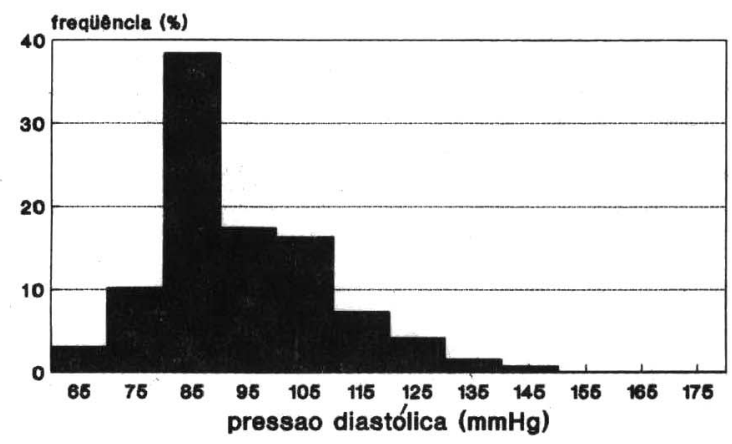

Figura 2. Distribulção de frequêncla da varıável tempo de trabalho como condutor geral (TCG) na população estudada.

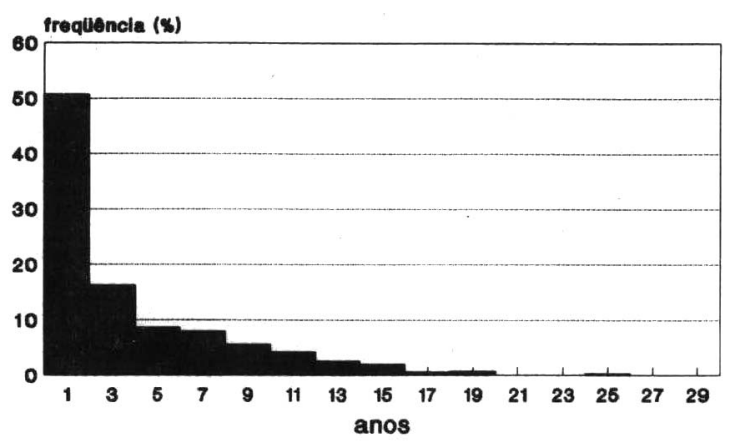

Figura 3. Distnbuição de frequência da varıavel tempo de trabalho como condutor urbano (TCU) na população estudada.

Para verificar a possibilidade de associação entre tempo acumulado de trabalho (TCG, TCU, TCF) e HAS, foi necessário contornar o obstáculo de que na medida em que aumenta o tempo acumulado de trabalho, aumenta também a idade de uma população. Em outras palavras, os trabalhadores com maior tempo acumulado de trabalho - em uma empresa, em uma ocupação, em uma cidade, entre outras - são os que têm maiores idades, existindo uma franca associação entre tempo acumulado de trabalho e idade, istoé, numa dada

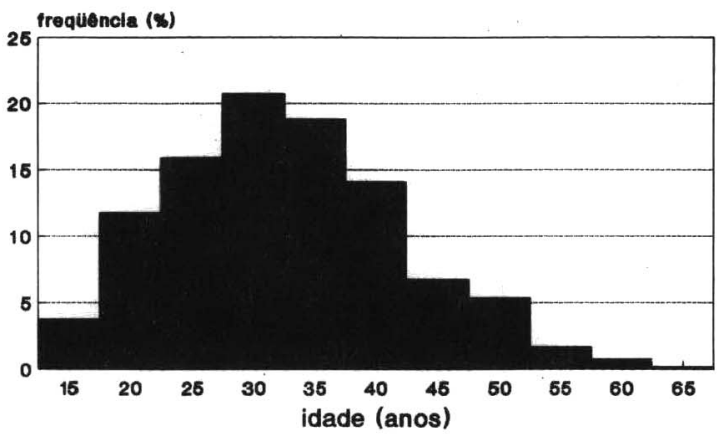

Figura 4. Distribulção de frequêncla da varıável rempo de trabalho como condutor frete (TCF) na população estudada.

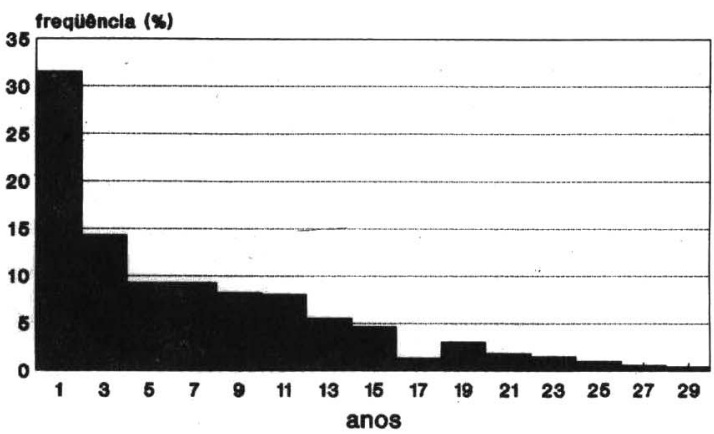

Figura 5. Distrıbuıção de frequêencia da pressão dıastólıca na população estudada.

população, os trabalhadores mais velhos são os que mais tempo trabalharam. A Figura 6 ilustra este fato, representando a distribuição conjunta das variáveis TCG e idade (Id) na população estudada. Observa-se tendência a aumento da variável TCG na medida em que aumenta a variável Id, sumariada pela reta de regressão daquela sobre esta.

Por outro lado, é conhecida a associação entre HAS e Id (Haddad ${ }^{8}$, 1967). Então, como isolar uma eventual associação entre tempo acumulado de trabalho e PAd da provável associação entre PAd e Id?

A opção metodológica feita foi utilizar um tratamento estatístico que possibilitasse verificar a existência de associação entre níveis pressóricos e tempo acumulado de trabalho, independentemente da variável Id. A escolha recaiu sobre a técnica de regressão linear múltipla* - tendo como variáveis independentes a Id e o tempo acumulado de trabalho, e variável dependente aPAd - que possibilitou o isolamento e identificação da

* Regressão linear múltipla é parte integrante de um conjunto de métodos estatísticos conhecido como "análise de regressão", que trabalha com a construção de modelos matemáticos que descrevemas relações que se estabelecementre uma variável dependente e um conjunto pré-estabelecido de variáveis independentes. 


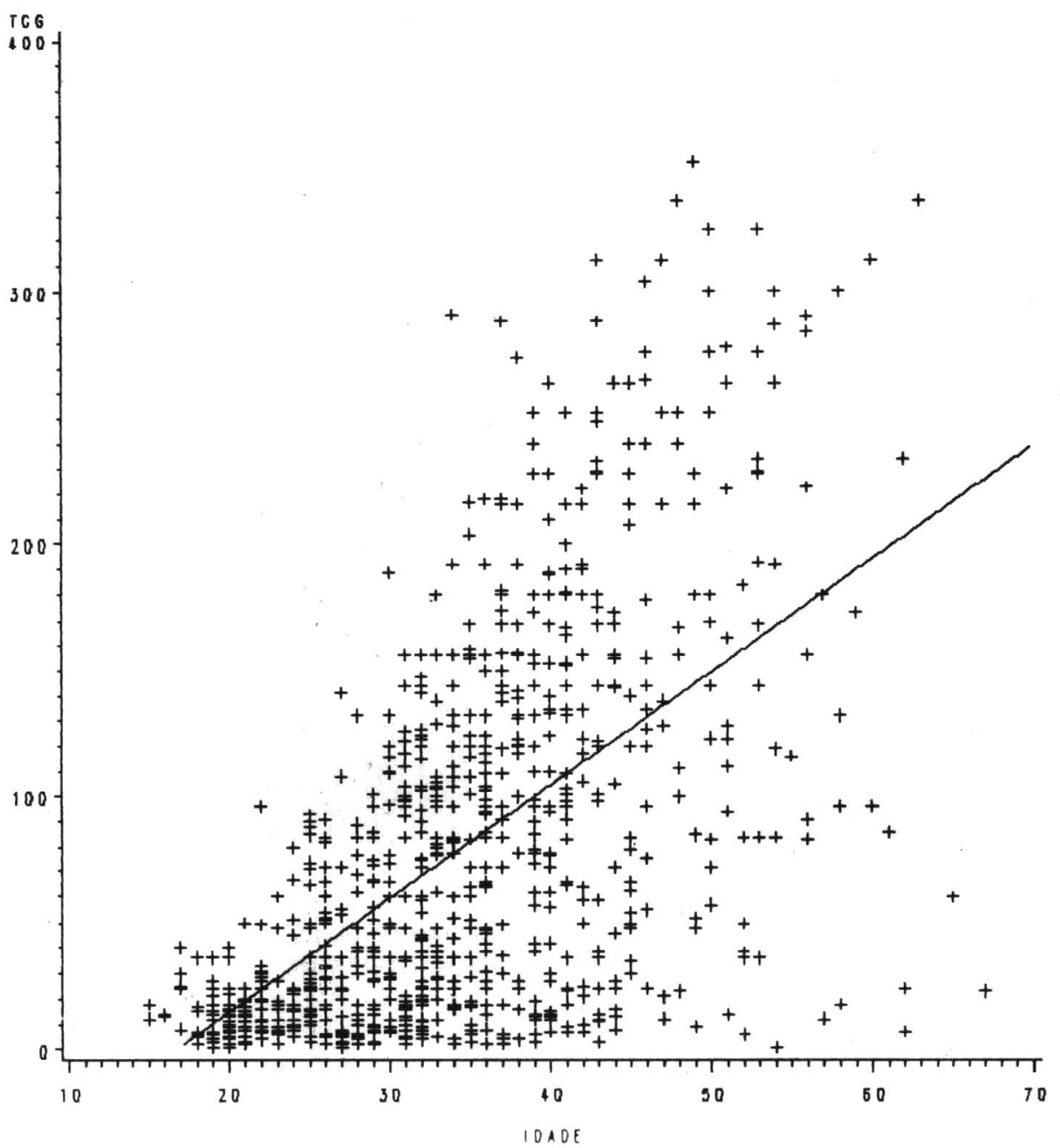

TCG medida em meses e IDADE medida em anos

Figura 6. Distribuição conjunta das varıávels tempo de trabalho como condutor geral (TCG) e idade.

influência da Id e da TCG (ou TCU, ou TCF) sobre a PAd, bem como o efeito da interação entre estas variáveis sobre a PAd.

Deste modo, o modelo utilizado foi:

$$
P A d_{3}=\alpha+\beta_{1} I d_{1}+\beta_{2} \ln \left(T C G_{1}\right)+\beta_{3} I d_{1} \cdot \ln \left(T C G_{i}\right)+\varepsilon_{1}
$$

onde a variável PAd é explicada através de uma combinação linear da variável Id e do logaritmo natural* da variável TCG, bem como da interação entre este e a Id.

Através da técnica de regressão linear múltipla ajustou-se o modelo:

$\widehat{P A d_{1}}=a+b_{1} I d_{1}+b_{2} \ln \left(T C G_{1}\right)+b_{3} I d_{t} \ln \left(T C G_{i}\right)$ onde os coeficientes $a, b_{1}, b_{2} e b_{3}$ estimulam os parâmetros $a, \beta_{1}, \beta_{2}$, e $\beta_{3}$.

* Aplicou-se uma transformação logarítmica à variável TCG como recurso para reduzir sua dispersão e aproximá-la da distribuição normal. Conforme se observa na Figura 2, a distribuição da TCG na população estudada é bastante assimétrica, lembrando uma distribuição exponencial, com mediana ( 54 meses) bastante a esquerda da média $(79,90$ meses). Além disso, esta distribuição apresenta grande dispersão (variância $=5.750,18$ meses $^{2}$ ). A Figura 7 mostra a distribuição da variável TCG transformada (logaritmo natural de TCG). De fato, após a transformação logarítmica da TCG obtém-se uma reduçāo drástica da dispersão dos dados (variância $=1.61\left[\ln (\text { mês) }]^{2}\right)$, e uma aproximação da simetria, com mediana $(3,99 \ln$ [mês]) bastante próxima da média (3.78 $\ln [\mathrm{mês}])$. 


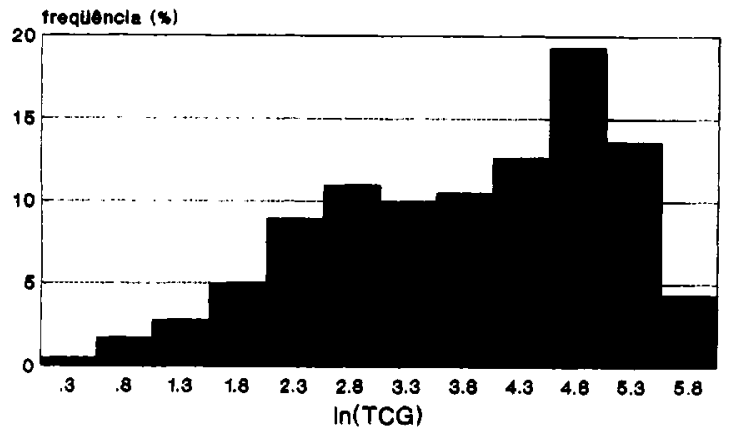

Figura 7. Distribuiçäo de frequência de In (TCG) na popula. ção estudada.

\section{Resultados}

A Tabela 1 resume os resultados obtidos na aplicação do modelo utilizado.

A Tabela 2 resume os resultados obtidos na aplicação de um modelo semelhante ao anteriormente utilizado, porém utilizando a variável TCU comoquantificadora do tempo acumulado de trabalho como condutor urbano.

A Tabela 3 resume os resultados obtidos na aplicação de um modelo semelhante ao anteriormente utilizado, porém utilizando a variável TCF como quantificadora do tempo acumulado de trabalho como condutor frete.

Sob os modelos de regressão utilizados, pode-se afirmar, para a população estudada:

1) Quanto ao modelo representado pela equação (2)

a) a análise de variância associada ao modelo representado pela equação (2) mostrou uma estatística F suficientemente grande para implicar num nível de significância da ordem de 0,0001 ;

b) a análise dos resíduos* mostrou que estes têm distribuição próxima da distribuição normal, além de dispersão razoavelmente uniforme quando "plotados"contra Id e contra ln(TCG);

* Entende-se por resíduos da regressão as diferenças entre os valores observados da variável dependente e os seus valores preditos pelo modelo proposto. A análise dos resíduos é um passo fundamental para o julgamento da qualidade do modelo. Este para estar bem ajustado aos dados deve produzir resíduos normalmente distribuídos, com média bastante próxima de zero.

** Que pressupõe:

a) PAd é variável aleatória observável.

b) Id e TCG são variáveis não aleatórias.

c) $\varepsilon$ évariável aleatória não observável e $a, \beta_{1}, \beta_{2}$ e $\beta_{3}$ sãoparâmetros desconhecidos $\left(a, \beta_{1}, \beta_{2}\right.$, e $\left.\beta_{3} \varepsilon R\right)$.

d) $\varepsilon_{1}, \varepsilon_{2}, \ldots,\left(\varepsilon_{83}\right)$ são estatisticamente independentes.

e) $\mathrm{E}\left(\varepsilon_{1}\right),=\mathrm{E}\left(\varepsilon_{2}\right), \ldots,=\mathrm{E}\left(\varepsilon_{839}\right)=$

f) $\mathrm{V}(\mathrm{E})=$ constante, desconhecida c) são válidos os pressupostos do modelo de regressão múltipla**;

d) levandoem consideração as estimativas e estatísticas apresentadas na Tabela 1, pode-se dizer que os dados suportam a expressão:

$\widehat{P A d_{i}}=59,76+0,78 I d_{i}+3,59 \ln \left(T C G_{1}\right)-0,08 I d_{i}, \ln \left(T C G_{i}\right)(5)$

(PAd em mmHg, Id em anos e TCG em meses)

2) Quanto ao modelo representado pela equação (3)

a) a análise de variância associada ao modelo representado pela equação (3) mostrou uma estatística F suficientemente grande para implicar num nível de significância da ordem de 0,0001 ;

b) a análise dos resíduos mostrou que estes têm distribuição próxima da distribuição normal, além de dispersão razoavelmente uniforme quando "plotados" contra a Id e contra $\ln (\mathrm{TCU})$;

c) são válidos os pressupostos do modelo de regressão múltipla;

d) levando em consideração as estimativas e estatísticas apresentadas na Tabela 2, pode-se dizer que os dados suportam a expressão:

$P A d_{1}=59,58+0,82 I d_{1}+3,61 \ln \left(T C U_{1}\right)-0,09 I d_{1} \cdot \ln \left(T C U_{1}\right)(6)$ (PAd em mmHg, Id em anos e TCU em meses)

\section{3) Quanto ao modelo representado pela equação(4)}

O modelo representado pela equação (4) não foi capaz de produzir estimativas significativas para os coeficientes de variação đas variáveis Ide $\ln (\mathrm{TCF})$, bem como para a interação entre elas. Em outras palavras, para o conjunto de dados obtidos com a população estudada, não se consegue associar, com um bom nível de significância, a variável PAdàs variáveis Ide $\ln (\mathrm{TCF})$.

\section{Discussáo}

As equações (5) e (6) evidenciam uma associação positiva entre PAd e Id, confirmando uma expectativa já bem estabelecida na literatura especializada. De fato, segundo tais equações, mantendo-se constante a variável TCG (ou a variável TCU, no caso da equação (6)), a PAd aumenta na medida em que aumenta a Id. No entanto, o que chama a atenção é que este acréscimo da PAd em função da Id̉ não é constante, mas sua grandeza depende da magnitude do tempo acumulado (TCG ou TCU) envolvido. Tal comportamento é o reflexo da interação existente entre as variáveis Id e TCG, bem como entre aquela e a TCU, expressa nas equaçoes (5) e (6), respectivamente, pelos termos:

$$
-0,08 I d_{i} \cdot \ln \left(T C G_{i}\right) \quad \text { e } \quad-0,09 I d_{i} \cdot \ln \left(T C U_{i}\right)
$$

Assim, segundo o modelo ajustado, o acréscimo da pressão arterial diastólica, associado a um dado acréscimo da idade, é maior para trabalhadores mais 
Tabela 1. Estimativas e estatísticas obtidas com a aplicação do modelo de regressão:

$$
\widehat{P A d_{i}}=a+b_{1} I d_{i}+b_{2} \ln \left(T C G_{i}\right)+b_{3} I d_{i} \cdot \ln \left(T C G_{i}\right)
$$

\begin{tabular}{ccccc}
\hline Parâmetro & \multicolumn{1}{c}{ Estimativa } & Erro Padrão & Valor de "t" p/Ho: Parâmetro=0 & "p-value" \\
\hline a & $59,76 \mathrm{mmHg}$ & $6,27 \mathrm{mmHg}$ & 9,531 & $<0,0001$ \\
$\beta_{1}$ & $0,78 \mathrm{mmHg} / \mathrm{ano}$ & $0,19 \mathrm{mmHg} / \mathrm{ano}$ & 4,010 & 0,0001 \\
$\beta_{2}$ & $3,59 \mathrm{mmHg} / \mathrm{mês}$ & $1,70 \mathrm{mmHg} / \mathrm{mês}$ & 2,117 & 0,0346 \\
$\beta_{3}$ & $-0,08 \mathrm{mmHg} /$ ano.mês & $0,05 \mathrm{mmHg} / \mathrm{ano}$.mès & $-1,702$ & 0,0891 \\
\hline
\end{tabular}

Tabela 2. Estimativas e estatísticas obtidas com a aplicação do modelo de regressão:

$$
\widehat{P A d_{i}}=a+b_{1} I d_{i}+b_{2} \ln \left(T C U_{i}\right)+b_{3} I d_{1} \cdot \ln \left(T C U_{i}\right)
$$

\begin{tabular}{ccccc}
\hline Parâmetro & Estimativa & Erro Padrão & Valor de "t" $p / H_{0}: P a r a ̂ m e t r o=0$ & "p-value" \\
\hline$a$ & $59,58 \mathrm{mmHg}$ & $6,47 \mathrm{mmHg}$ & 9,210 & 0,0001 \\
$\beta_{1}$ & $0,82 \mathrm{mmHg} / \mathrm{ano}$ & $0,19 \mathrm{mmHg} / \mathrm{ano}$ & 4,268 & 0,0001 \\
$\beta_{2}$ & $3,62 \mathrm{mmHg} / \mathrm{mês}$ & $1,98 \mathrm{mmHg} / \mathrm{mês}$ & 1,826 & 0,0682 \\
$\beta_{3}$ & $-0,09 \mathrm{mmHg} /$ ano.mês & $0,05 \mathrm{mmHg} / \mathrm{ano}$.mês & $-1,654$ & 0,0986 \\
\hline
\end{tabular}

Tabela 3. Estimativas e estatísticas obtidas com a aplicação do modelo de regressão:

$$
\widehat{P A d}_{i}=a+b_{1} I d_{i}+b_{2} \ln \left(T C F_{i}\right)+b_{3} I d_{i} \cdot \ln \left(T C F_{i}\right)
$$

\begin{tabular}{ccccc}
\hline Parâmetro & Estimativa & Erro Padrão & Valor de "t"p/Ho :Parâmetro=0 & "p-value" \\
\hline a & $58,55 \mathrm{mmHg}$ & $17,74 \mathrm{mmHg}$ & 3,301 & 0,0011 \\
$\beta_{1}$ & $0,75 \mathrm{mmHg} / \mathrm{ano}$ & $0,46 \mathrm{mmHg} / \mathrm{ano}$ & 1,627 & 0,1047 \\
$\beta_{2}$ & $4,17 \mathrm{mmHg} / \mathrm{mês}$ & $4,36 \mathrm{mmHg} / \mathrm{mês}$ & 0,955 & 0,3400 \\
$\beta_{3}$ & $-0,08 \mathrm{mmHg} /$ ano.mês & $0,11 \mathrm{mmHg} / \mathrm{ano}$.mês & $-0,750$ & 0,4540 \\
\hline
\end{tabular}

jovens na profissão do que para aqueles com maior experiência profissional.

Tomando-se como exemplo a equação de regressão ajustada para as variáveis preditoras Id e TCG, equação (2), pode-se deduzir o acréscimo na pressão arterial diastólica entre os instantes 2 e 1 como:

$\Delta P A d=0,78\left(I d_{2}-I d_{1}\right)+3,59 \ln \left[\frac{T C G_{2}}{T C G_{1}}\right]-0,08 \ln \left[\frac{\left(T C G_{2}\right)^{l d_{2}}}{\left(T C G_{1}\right)^{I d_{1}}}\right](7)$

Analisando-se a expressão acima percebe-se que, mantendo-se constante a diferença entre os valores $\mathrm{TCG}_{2}$ e TCG (tempo acumulado de trabalho como condutor geral nos instantes 2 e 1 ), diferença esta que baliza o "envelhecimento profissional" do condutor, o acréscimo na PAd associado ao acréscimo de Id, "envelhecimento biologico", diminui quando as faixas de tempo de trabalho aumentam.
Exemplificando, um trabalhador que aos 25 anos tem TCG igual a 24 meses, após trabalhar 10 anos como condutor passa a ter TCG igual a 144 meses e Id igual a 35 anos. Segundo a equação (7), o acréscimo predito em sua pressão arterial diastólica é de $6,67 \mathrm{mmHg}$. No entanto, se este trabalhador tivesse aos 25 anos TCGigual a 120 meses (enão 24 mesescomo no casoanterior), após trabalhar os mesmos 10 anos teria também Id igual a 35 anos mas TCG igual a 240 meses. Neste caso, o acréscimona variávelPAd seria de $4,52 \mathrm{mmHg}$. Percebe-se que o acréscimo da PAd predito para o trabalhador em início de carreira excedeu em $48 \%$ aquele predito para o trabalhador mais velho na profissão, muito embora o tempo transcorrido entre $\mathrm{TCG}_{1}$ e $\mathrm{TCG}_{2}$ e as idades tenha sido exatamente o mesmo nos dois casos.

Analogamente, na amplitude dos dados aqui trabalhados, observa-se uma associação positiva entre a variável dependente PAd e a variável preditora TCG (e 
TCU). Isto é, observa-se que, independentemente da variável idade, a PAd aumenta na medida em que aumenta o tempo acumulado de trabalho, quantificado quer pela TCG, quer pela TCU. Semelhantemente ao que ocorre na associação entre PAd e Id, também a associaçãoentre PAde TCG (ou TCU) nãoé linear, mas sim é modulada pela interação existente entre as variáveis TCG (e TCU) e Id. Esta interação faz com que a magnitude da variação da PAd em função da variável TCG (ou TCU), dependa também da faixa em que varia a idade do condutor.

Assim, segundo o modelo ajustado, o acréscimo da pressão arterial diastólica associado a um acréscimo constante da TCG (ou TCU) é maior para os trabalhadores mais jovens do que para aqueles mais velhos.

Exemplificando, um trabalhador que aos 20 anos tem TCG igual a 24 meses, após trabalhar 10 anos nesta profissão passa a ter TCG igual a 144 meses e Id igual a 30 anos. Segundo a equação (7), o acréscimo predito em sua pressão arterial diastólica é de $7,39 \mathrm{mmHg}$. No entanto, se este trabalhador tivesse TCG igual a 24 meses aos 40 anos (e não aos 20 como no caso anterior), após trabalhar os mesmos 10 anos teria também TCG igual a 144 meses mas Id igual a 50 anos. Neste caso, o acréscimo na variável PAd seria de $4.52 \mathrm{mmHg}$. Percebe-se que o acréscimo da PAd predito para o trabalhador mais jovem foi $63 \%$ maior que aquele predito para o mais velho, muito embora o tempo trabalhado e o acréscimo na idade tenham sido exatamente os mesmos nos dois casos.

A Figura 8 ilustra o que foi aqui discutido. Ela reproduz a variação do valor predito para a PAd em função das variáveis Id e TCG, segundo a equação (7). Observa-se que na medida em que a Id vai aumentando,

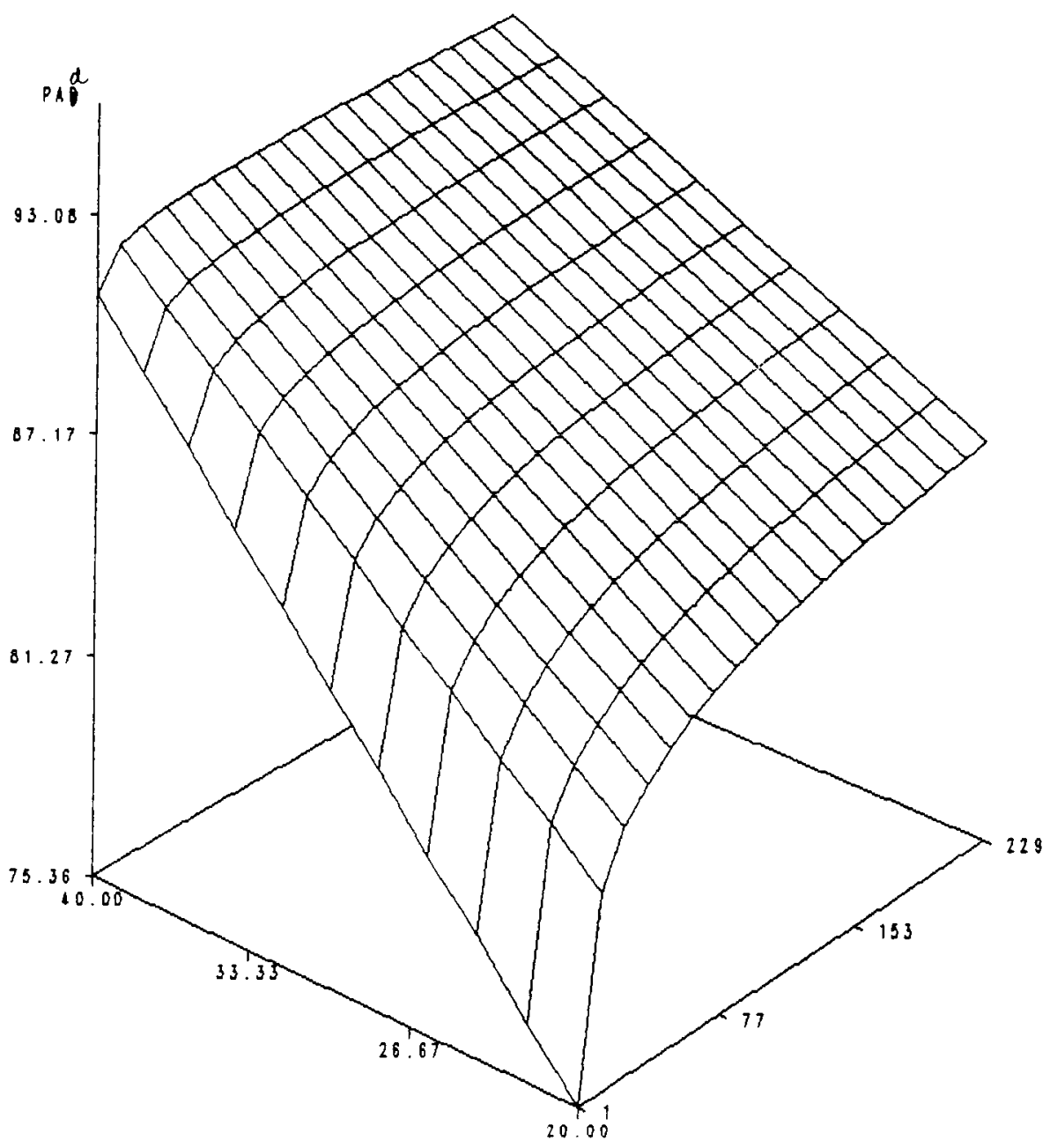

PAd (mmHq), IDADE (anos), TCG (meses)

Figura 8. Varıaçào do valor predıto para a pressào arterıal diastliıca (PAd) em função das variações idade (Id) e iempo de trabaho como condutor geral (TCG). 
os aumentos da PAd associados a aumentos da TCG vão diminuindo, isto é, em idades menores a variação da PAd em função da TCG é maior do que a ocorrida em idades maiores. Ou então, visto sob outra óptica, observa-se que o aumento da PAd associado ao aumento da Id diminui na medida em que aumenta a TCG.

Outra importante observação sobre a Figura 8 é que, mantendo-se a Id do trabalhador constante, o aumento instantâneo da PAd em função de um aumento da TCG vai diminuindo na medida em que a faixa onde ocorre esta variação aumenta, reflexo do "efeito logarítimico" da variável TCG sobre a variável PAd.

Resultados semelhantes se obtêm quando se utiliza a variável TCU ao invés da TCG.

Na interpretação desses achados algumas hipóteses podem ser levantadas. A primeira delas seria que os dados refletem o efeito da "evasão" do trabalho daqueles trabalhadores que apresentaram alterações pressóricas (que se sabe ocorrem acompanhadas de outras alterações de saúde) na medida em que foram exercendo sua profissão. Tal fenômeno conhecido como "efeitodo trabalhador sadio" (McMichael e col. ${ }^{17}$, 1975), se dá quer por incapacidade própria do trabalhador em manter-se em atividade (devido a adoecimento ou morte), quer por interferência direta dos serviços de saúde das empresas, que selecionam os mais "hígidos" para o trabalho. Na população geral a prevalência da HAS aumenta na medida em que se passa dos grupos mais jovens para os de mais idade, atingindo-se um patamar na década dos 60 anos (Haddad ${ }^{8}$ 1967). $\mathrm{Na}$ população estudada, ao contrário, a maior prevalência da HAS * foi encontrada já na faixa dos 40 a 49 anos. estabilizando-se a partir de então. A Tabela 4 ilustra 0 presente comentário.

* Os trabalhadores da população estudada foram classificados quanto à pressão arterial em dois grupos mutuamente exclusivos: normotensos e hipertensos. O critério diagnóstico utilizado para a classificação como "normotenso" foi: apresentar pressão diastólica menor que $100 \mathrm{mmHg}$ na primeira avaliação clínica no PST, na ausência de medicação antihipertensiva. O critério diagnóstico para "hipertenso" foi:

a) apresentar pressão diastólica maior ou igual a $120 \mathrm{mmHg}$ na primeira avaliação clínica no PST, ou

b) apresentar pressão diastólica entre 110 e 115 nas duas primeiras avaliações clínicas no PST, ou

c) apresentar pressão diastólica maior ou igual a $100 \mathrm{~mm} H g$ nas três primeiras avaliações clínicas no PST, ou

d) apresentar pressão diastólica menor entre 100 e $105 \mathrm{mmHg}$ sob uso de medicação anti-hipertensiva regulamente há no mínimo um mês.

** Sabe-se que existe associação positiva entre exposição crônica excessiva a ruídoe HAS (Parvizpoor ${ }^{19}$, 1979; Jonsson \& Hansson ${ }^{9}$. 1977; Manninen \& Aro ${ }^{16}$, 1979; Fouriad e col. ${ }^{6}, 1984$; Talbott e col. ${ }^{23}$, 1985; Wu e col..24; 1987; Kristensen ${ }^{13}, 1989$ ).
Tabela 4. Prevalência da HAS na população estudada, dividida em faixas etárias de 10 anos.

\begin{tabular}{ccc}
\hline Idade (anos) & Prevalência (\%) & d.p. \\
\hline 20 a 29 & 10,3 & 1,02 \\
30 a 39 & 33,1 & 1,33 \\
40 a 49 & 40,6 & 1,89 \\
50 a 59 & 37,3 & 3,21 \\
mais de 59 & 37,7 & 8,47 \\
\hline
\end{tabular}

Os dados da Tabela 4 são compatíveis com a idéia de que pode estar havendo uma "evasão" dos condutores hipertensos para a população geral.

Outra hipótese seria a melhoria das condições de trabalho, particularmente as relacionadas à diminuição da jomada de trabalho e à diminuição do ruído produzido pelos ônibus**, que vêm ocorrendo nos últimos anos, o que tomaria o exercício da profissão menos "patogênico" em anos recentes.

Outra hipótese ainda que pode ajudar na interpretação do achado mostrando diferenças quanto à associação entre PAd e TCG (ou TCU) entre trabalhadores mais jovens e mais velhos na população estudada se prende à possibilidade de o impacto do exercício da profissão, com as características com as quais ela se deu para este grupo em particular, ser maior nos primeiros meses e anos (onde, por hipótese, as alterações provocadas pelos novos hábitos e ritmos de trabalho provocariam maiores efeitos) do que posteriormente (quando os trabalhadores teriam criado estratégias diferenciais de trabalho, que ajudariam na absorção e acomodação do impacto sofrido).

Os achados aqui apresentados encontram eco na literatura especializada. Ribeiro ${ }^{20}$ (1983), em estudo seccional realizado na cidade de São Paulo, estudou a distribuição da pressão arterial de mulheres trabalhadoras oriundas de diversos setores da economia, analisando a interferência de algumas variáveis, dentre elas algumas mais diretamente ligadas à ocupação, tais como setor de economia, tipo de trabalho e extensão diária e semanal da jornada. Essa pesquisa identificou associação entre o número de horas trabalhadas e a pressão diastólica, independentemente de outras variáveis, isto é, a média das pressões diastólicas das trabalhadoras, cujo total de horas trabalhadas por semana era menor que a mediana desta distribuição, era menor que a média dos outros $50 \%$ da população. O mesmo acontecia quando se tomava como parâmetro o número diário de horas trabalhadas. A média das pressões diastólicas entre aquelas que trabalhavam até uma hora por dia era menor que a daquelas que trabalhavam entre 1 e 5 horas por dia, que por sua vez era menor que a daquelas que trabalhavam mais do que 5 horas por dia.

Os achados aqui discutidos corroboram esta tendência, na medida em que se firmou uma associação 
positiva entre PAd e o tempo de trabalho, tomado logaritmicamente, na categoria de condutor.

Tal associação caracteriza-se quando se utiliza o tempo de trabalho como motorista urbano (TCU), mas não se estabelece quando este tempo é quantificado especificamente para o trabalho como motorista de coletivos fretados (TCF).

Sintomaticamente, os motoristas consensualmente apontam o trabalho nos veículos coletivos de transporte urbano como a pior opção que a eles se apresenta. Os motivos levantados são os mais variados: más condições de manutenção dos ônibus, implicando em grande nível de barulho produzido pelo motor e maior esforço muscular para conduzir o veículo; más condições do tráfego urbano; atritos constantes com os usuários do serviço; atritos constantes com os fiscais de terminal e a chefia; horários invariavelmente exíguos a serem cumpridos; descanso insuficiente dentro da jornada de trabalho; refeiçðes realizadas fora do horário habitual e em tempo reduzido; menores salários; trabalho em turnos.

Nesse sentido, é plausível supor que a maior taxa de variação daPAd em funçãodo tempode trabalho seja encontrada quando este é quantificado particularmente pela TCU. Tal achado vem ao encontro a uma expectativa, por parte da categoria dos condutores, no sentido de caracterizar-se uma maior "patogenicidade" associada ao exercício da profissão enquanto "urbano" em relação à modalidade "frete".

\section{Consideraçóes finais}

Concluindo, através do modelo de regressão múltipla aqui utilizado, firmou-se uma associação positiva entre pressão arterial diastólica e idade, fatoeste bastante conhecido da literatura especializada. Além disso, outro achado, este ausente das discussðes especializadas, foi a associação positiva encontrada entre pressão arterial diastólica e tempo acumulado de trabalho, que chega a ter um caráter dose-resposta.

Também importante foi a interação firmada entre idade e tempo acumulado de trabalho, que modula as duas associações acima referidas.

Tais achados se restringem à população estudada, e sua generalização para a população de referência é bastante dificultada - além dos problemas inerentes a esta extrapolação - pelas limitações próprias do presente estudo: utilização de informação secundária, ausência no modelo ajustado de covariáveis importantes e desenho epidemiológico transversal, com população não aleatorizada.

Por trás do desenvolvimento do presente estudo esteve a seguinte indagação: o trabalho como condutor causa hipertensão? A discussão aqui apresentada, evidentemente, está longe de estabelecer qualquer relação de causa e efeito entre trabalho e hipertensão.
Mesmo uma associação entre trabalho como condutor e hipertensão está longe de ser aqui firmada para os trabalhadores em geral. Entretanto, os achados encorajam tal busca, onde certamente é necessária a construção de um instrumental metodológico mais potente, superando as limitaçð̌es aqui evidenciadas.

\section{Agradecimento}

Ao Laboratório de Aplicação em Epidemiologia (LAPE), do Departamento de Medicina Preventiva e Social daFaculdade de Ciências Médicas daUNICAMP, pelo apoio na elaboração do presente estudo.

CORDEIRO, R. et al. [Diastolic blood pressure and its association with cumulative working time among urban bus workers]. Rev. Saúde Pública, 27:363-72, 1993. The relationship between diastolic blood pressure and the variables "total cumulative working time" and "age" was examined by regression analysis. The study was carried out among 839 bus drivers and conductors, users of an occupational heal th center in Campinas, S.Paulo State, Brazil. The main results were a positive association between diastolic blood pressure and cumulative working time, as well as an interaction between this variable and the bus worker's age.

Keywords: Blood pressure. Transportation. Workers.

\section{Referências Bibliográficas}

1. CARTER, N.L. Eye colour and susceptibility to noise-induced permanent thereshold shift. Audiology, 19: 86-93, 1980.

2. COBB, S. \& ROSE, R.M. Hypertension, peptic ulcer, and diabets in air traffic controllers. JAMA, 224: 489-92, 1973.

3. CORDEIRO, R. Pressão arterial diastólica entre motoristas e cobradores de Campinas, usuários de um seriço de saúde ocupacional. Campinas, 1991. [Dissertação de Mestrado Faculdade de Ciências Médicas da UNICAMP].

4. COTTINGTON, E. et al. Occupational stress and diastolic blood pressure in a blue-collar population: the Pittsurgh noise-hypertension project. Am J.Epidemiol., 118: 440-6, 1983.

5. DRETTENER, B. et al. Cardiovascular risk factors and hearing loss - a study of 1000 fifty-years-old men. Acta Otolaryngol, 79: 366-71, 1975.

6. FOURIAD, $\mathrm{C}$. et al. Influence of socioprofessional conditions on blood pressure levels and hypertension control. Am.I.Epidemiol., 120: 72-86, 1984.

7. GARDELL, B. et al. The working environment for local public transport personel: report. Estocolmor. Swedish Work Environment Fundation, 1977.

8. HADDAD, N. Inquérito epidemiológico sobre cardiopatias crônicas em um bairro de Ribeirão Preto, São Paulo, Brasil. Arq.Hig.Salide Ptiblica, 32/33 (111/118): 27-77, $1967 /$ 1968.

9. JONSSON, A. \& HANSSON, L. Prolonged expsure to a stressfull stimulus (noise) as a cause of raised blood pressure in man. Lancet, 1: 86-7, 1977.

10. KARASEK, R.A. Job decision latitude, job demands, and cardio-vascular disease: a prospective study of Swedish men. AmJ.Public Health, 71: 694-705, 1981. 
11. KARASEK, R.A. et al. Job demands, job decision latitude, and mental strain: implications for a job redesign. Adm.Sci.Q., 24: 285-308, 1979.

12. KARASEK, R.A. et al. Job psychological factors and coronary heart disease. Adv.Cardiol., 29: 62-7, 1982.

13. KRISTENSEN, T.S. Cardiovascular disease and the work environment - a critical review of the epidemiologic literature on nonchemical factors. Scand J. Wk Environ. Health, 15: $165-79,1989$.

14. LAURELL, A.C. \& NORIEGA, M.Trabajoy saluden Sicartsa. Ciudad de México, Programa de Difusión Cultural del SITUAM, 1987.

15. MACKIE, R.R. \& O'HANLON, J.L. A study of the combined effects of extended driving and heat stress on driver arousal and performance. In: Mackie, R.R., ed. Vigilance: theory, operatinal performance and physiological correlates. New York. Human Factors Plenum Press, 1977. (NATO Conference Series III) p. 537-58.

16. MANNINEN, O.\& ARO, S. Noise-induced hearing loss and blood pressure. Int.Arch.Occup Environ.Health, 42: 2516, 1979.

17. McMICHAEL, A.J. et al. Observations on the evalution of occupational mortality data. J.Occup.Med., 17: 128-31, 1975.
18. NETTERSTROM, B. \& JUEL, K. Impact of work-related and psychosocial factors on the development of ischemic heart disease among urban bus drivers in Denmark. Scand J.WkEnviron. Health, 14: 231-8, 1988.

19. PARVIZPOOR, D. Noise-induced hearing loss and blood pressure.Int.Arch.Occup.Environ.Health, 42: 251-6, 1979.

20. RIBEIRO, M.B.D. Hypertension among female workers in São Paulo, Brazil: predictors and joint effects. Hypertension, 5(6): V144 - V148, 1983.

21. RUSCONI, C. et al. Fattori di rischio coronarico e cardiopatia ischemica nei conduttori enei bigliettari di autobus. Minerva Cardiol., 23: 718-27, 1975.

22. SHEKELLE, R.B. et al. Social status and incidence of coronary heart disease. J.Chron.Dis., 22: 381-94, 1969.

23. TALBOTT, C. etal Occupational noise exposure, noise induced hearing loss, and the epidemiology of high blood pressure. Am.J.Epidemiol., 121: 501-14, 1985.

24. WU, T. et al. Study of noise exposure and high blood pressure in shipyard workers. Am J.Ind.Med., 12: 431-8, 1987.

Recebido para publicação em 12.3.1993 Aprovado para publicação em 7.6.1993. 